\title{
Data acquisition and digital filtering in analytical isotachophoresis
}

\section{J. C. REIJENGA}

Laboratory of Instrumental Analysis, Eindhoven University of Technology, P.O. Box 513, 5600 MB Eindhoven (The Netherlands)

\begin{abstract}
Voltage-to-frequency conversion (VFC) or 12-bit integrating analog-to-digital conversion with data bunching/averaging during $50 \mathrm{~ms}$ is suggested as the optimum data acquisition technique in isotachophoresis. Computer simulation of digital filtering of the conductivity and UV signal in analytical isotachophoresis in the time domain with Savitzky-Golay filtering, commonly used in chromatography, leads to unwanted oscillations at the stepwise change of the signal at the zone-to-zone boundary. If it is really necessary to remove noise prior to further signal evaluation, a rectangular moving average filter is preferred. In that case, a signal restoration algorithm should be applied to the filtered signal.
\end{abstract}

\section{INTRODUCTION}

The conductivity and UV detector signals in analytical isotachophoresis are characterized by a number of zones or plateaux of constant amplitude, separated by a more or less stepwise increase or decrease in time [1,2]. Disturbances can be of electronic, chemical or physical in nature, the origin of which is beyond the scope of this paper. It suffices to know that these disturbances can lead to either drift during detection of a zone, a distortion of the stepwise change or noise superimposed on the signal.

Plotting the signal on a potentiometric recorder is usually no problem, because of the time constant of the instrument ( $c a .0 .3 \mathrm{~s}$ ). Obviously, this time constant should not be so high as to disturb the stepwise changes of the signal. When computerized data acquisition is applied, analog-to-digital conversion (ADC) sampling frequencies in the range $10-50 \mathrm{~Hz}$ are mostly used [3-6]. If present, stochastic or periodic disturbances below this frequency range are also sampled. In this instance, a digital filter is applied. In recent publications on computerized data evaluation in isotachophoresis $[5,7]$ application of a time-domain filter according to the procedure of Savitzky and Golay [8] was described. In their original paper, Savitzky and Golay indicated that, for noise reduction in signals with Gaussian peaks, their filter performs better than exponential, triangular and (rectangular) moving average filters.

In isotachophoresis, the main criterion for the choice of filter type is that the 
stepwise change is not affected. This paper will show, through computer simulation of digital filtering, that of the time-domain filters mentioned the Savitzky-Golay type is less suitable. Whenever filtering is really necessary, a (rectangular) moving average (MA) type of filter is preferred.

\section{EXPERIMENTAL}

\section{Data acquisition}

The isotachophoretic signal contains both qualitative and quantitative information. In data acquisition, the associated parameters are resolution and sampling frequency, respectively. The reproducibility of the signal amplitude in present-day detectors (conductivity, potential gradient, UV absorption) does not require a resolution greater than 12 bits.

Capillary isotachophoresis is usually carried out at current densities that represent a compromise between speed of analysis and zone boundary disturbance due to convection and diffusion [9]. The resulting zone boundary profile gives rise to a zone transition of $c a .1 \mathrm{~s}$. In order to describe this zone transition, and thus determine accurately the location of the inflection point, sampling frequencies higher than $20 \mathrm{~Hz}$ are unnecessary. Otherwise, additional information (fluctuations), not originating from isotachophoretic phenomena, are also sampled. Moderate speed ADCs employ either of two principles of operation: successive approximation or integration. In the former instance, averaging times are typically $50 \mathrm{~ms}[10]$. These devices are thus overspecified by a factor 1000 at a sampling frequency of $20 \mathrm{~Hz}$. In addition, fluctuations in the range $20-20000 \mathrm{~Hz}$ can lead to aliasing. For data acquisition in chromatography, therefore, voltage-to-frequency convertors (VFCs) or integrating ADCs are preferred. Most chromatographic data acquisition stations are equipped with such devices, which combine integrating capacity with high resolution [11]. The former characteristics makes them especially useful for isotachophoresis.

\section{Simulation of digital filtering}

Computer simulation of digital filtering was performed with CLEOPATRA [12], modified and adapted [13] for use in a practical course for second-year students of the Chemical Engineering Faculty at our University. One of the modules of the program performs time-domain filter functions on signals generated by the program, or on data files. The latter can consist of real data measured by another program, with the important restriction that they consist of 256 data points only. For simulation and demonstration purposes, however, this poses no problems.

\section{Simulation of isotachophoretic signals}

In order to generate isotachophoretic signals, a computer program (ITPSIMUL) was written in Quickbasic (Microsoft, Redmond, WA, U.S.A.). A 256-point isotachopherogram could be generated, choosing the number of zones and their respective height and length. The stepwise signal increase from zone to zone is usually disturbed by a zone boundary profile due to convection and diffusion [1,9]. As an approximation, an eleven-point Gaussian-shaped disturbance was assumed and thus convoluted with the generated isotachopherogram. Finally, white noise could be added to the signal. The generation of such an isotachopherogram is illustrated in Fig. 1. 

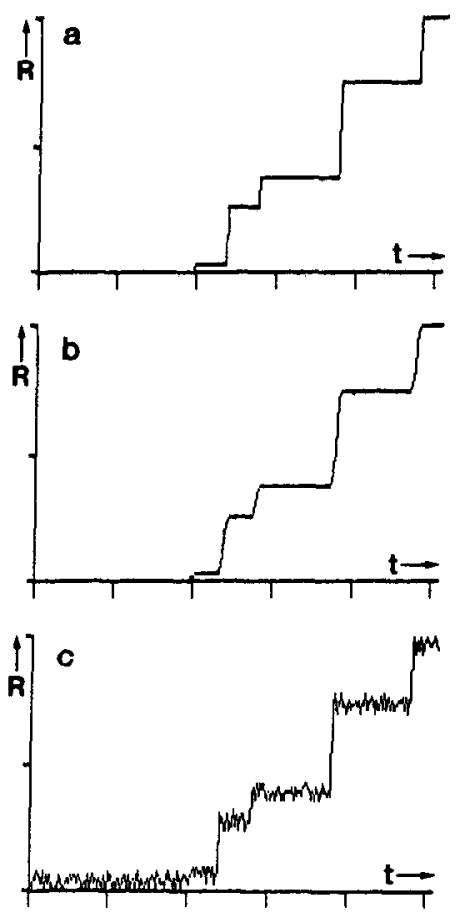

Fig. 1. Isotachopherogram, generated by the program ITPSIMUL, (a) consisting of a number of zones with infinitely sharp boundaries (b), after convolution with a Gaussian disturbance and (c) after addition of $5 \%$ white noise. $\mathrm{R}=$ Resistance; $\mathrm{t}=$ time.

The signals were stored in sequential files, compatible with the modified CLEOPATRA format.

\section{RESULTS AND DISCUSSION}

\section{Noise reduction}

A noisy baseline was generated and filtered with both a Savitzky-Golay (S-G) and a (rectangular) moving average (MA) digital filter with a filter width ranging between 5 and 21 points. The results are shown in Fig. 2. The MA-type filter is always more effective in noise reduction, although the net gain is only $30 \%$ at higher filter widths. However, one should realize that the results indicate that thirteen-point MA filter performs better than a $21-$ poiñ $\bar{S}-\mathbf{G}$ filter.

If using a conventional algorithm, an additional advantage of an MA filtcr is that the algorithm executes faster, because of the equal weighting factors. An $n$-point S-G filter requires $(n-1) / 2$ multiplications and $(3 n-3) / 2$ additions per data point, an MA filter 1 and $n$, respectively. Faster algorithms for these filters have also been developed [14].

\section{Signal distortion}

As mentioned above, the simulated isotachopherograms were convoluted with 


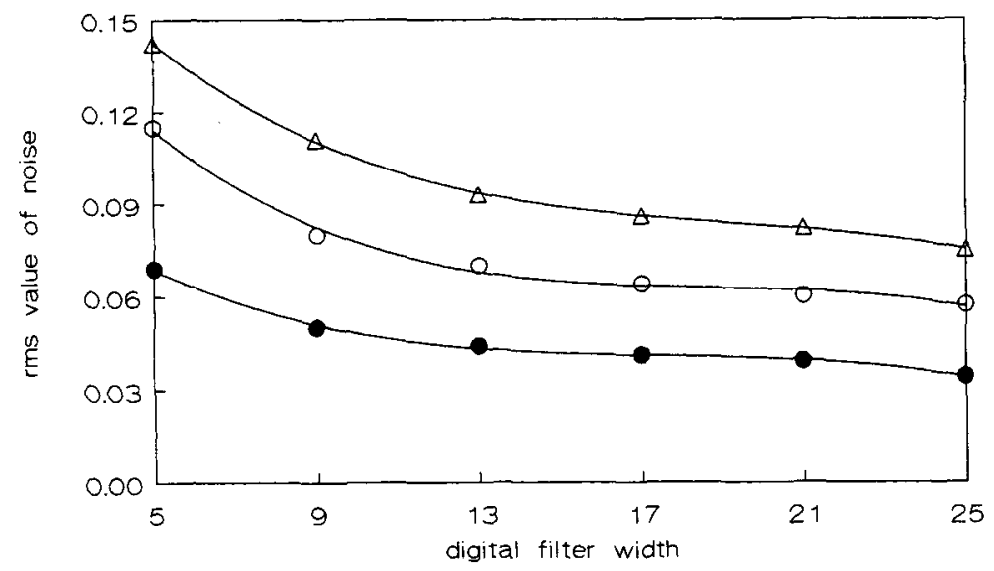

Fig. 2. Root-mean-square (rms) value of the noise on a straight baseline after applying a digital filter with different width. ( $\bigcirc)$ Savitzky-Golay, $(\bullet)$ rectangular moving average and $(\Delta)$ exponential filter. The rms value of the noise in the original signal was 0.161 .

an eleven-point Gaussian disturbance. In practice, a zone transition time of $1 \mathrm{~s}$ corresponds to a relatively sharp boundary, and is usually encountered only in optimized cationic separations. Thus, with a sampling frequency of $10-20 \mathrm{~Hz}$ an excellent description of the signal during the zone transition is obtained. There is obviously no
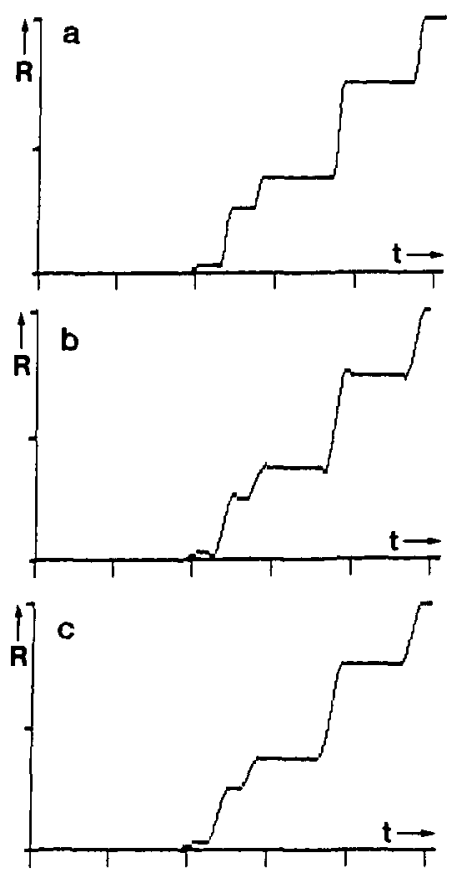

Fig. 3. Simulated isotachopherogram (a) filtered with (b) a seventeen-point cubic S G filter (c) a nine-point MA filter. These filters effect comparable degrees of noise reduction. 
signal distortion if the width of the MA filter is smaller than the number of data points during the zone transition.

For very noisy signals, e.g., from a UV detector at $206 \mathrm{~nm}$ [15], combined with sharp zone boundaries, a filter with a larger width may be necessary. In that event, signal distortion can take place. Fig. 3, illustrates the effect of both an $\mathrm{S}-\mathrm{G}$ and an MA filter. Noise has been omitted in order to show the difference between the two filters. It is seen that the $\mathrm{S}-\mathrm{G}$ filter introduces an oscillation, which gives two or more additional inflections points. This has severe consequences for the algorithm that determines the number and length of the zones in the filtered signal. An MA filter of comparable performance in terms of noise reduction does not introduce these oscillations, but instead causes a minor uncertainty in the exact location of the inflection point.

\section{Signal restoration}

To overcome the effects of possible distortion, a restoration algorithm was developed and applied to the filtered signal. The idea is to replace a filtered data point
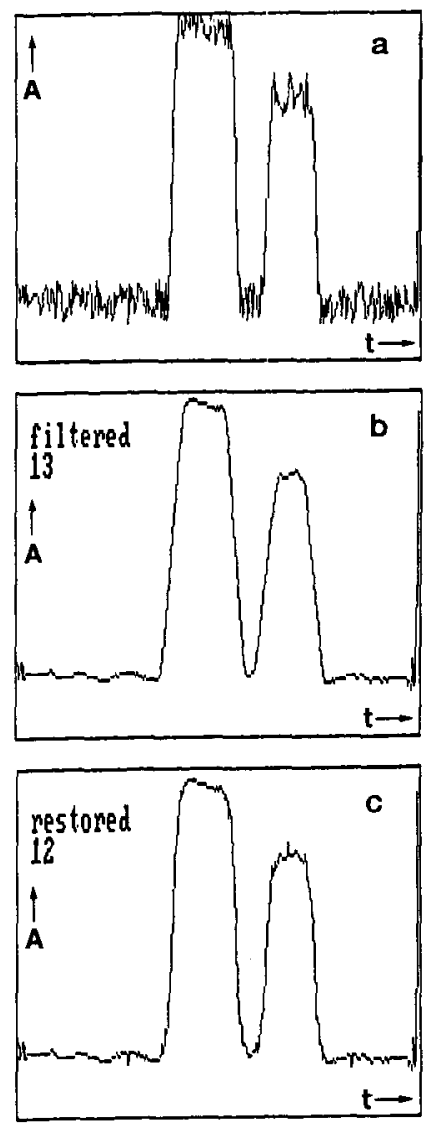

Fig. 4. Effect of the restoration algorithm on a filtered noisy signal. (a) Original signal filtered with (b) a thirteen-point MA filter and (c) restored. $\mathrm{A}=$ Absorbance. 
with the average of the filtered and the original data point, if the filtered signal deviates substantially from the original. To this end, the standard deviation of the difference between the filtered and the original baseline is determined first. A threshold value of twelve times this standard deviation is taken. The effect is illustrated in Fig. 4, a simulated very noisy UV signal. It can be seen that the restoration algorithm improves the zone boundary sharpness of the filtered signal at the cost of a slightly higher noise level. The execution time of this restoration algorithm is small compared with that of the MA filter. It is therefore advised to include this restoration algorithm in the smoothing routine.

\section{CONCLUSIONS}

Data acquisition using a VFC or integrating ADC with a 50-ms averaging time is recommended. Only for noisy signals should a rectangular moving average filter with a minimum width be used. In that event, a signal restoration algorithm should be applied to the filtered signal.

\section{REFERENCES}

1 F. M. Everaerts, J. L. Beckers and Th. P. E. M. Verheggen, Isotachophoresis-Theory, Instrumentation and Applications (Journal of Chromatography Library, vol. 6), Elsevier, Amsterdam, 1976.

2 P. Bocek, M. Deml, P. Gebauer and V. Dolnik, Analytical Isotachophoresis, VCH, Weinheim, 1988.

3 J. C. Reijenga, W. van Iersel, G. V. A. Aben, Th. P. E. M. Verheggen and F. M. Everaerts, J. Chromatogr., 292 (1984) 217.

4 J. C. Reijenga, Th. P. E. M. Verheggen and F. M. Everaerts, J. Chromatogr., 267 (1983) 75.

5 B. J. Wanders, Graduation Report, University of Technology, Eindhoven, 1988.

6 F. S. Stover, K. L. Deppermann and W. A. Grote, J. Chromatogr., 269 (1983) 198.

7 B. J. Wanders, A. A. G. Lemmens, M. M. Gladdines and F. M. Everaerts, J. Chromatogr., 470 (1989) 79.

8 A. Savitzky and M. J. E. Golay, Anal. Chem., 36 (1964) 1627

9 J. C. Reijenga, Th. P. E. M. Verheggen and F. M. Everaerts, J. Chromatogr., 328 (1985) 353.

10 Data Acquisition Handbook, Analog Devices, Norwood, MA, 1982.

11 Product Data Book, Burr Brown, Tucson, AZ, 1982.

12 G. Kateman, P. F. A. van der Wiel, T. H. A. M. Janse and B. G. M. Vandeginste, CLEOPATRA (Chemometrics Library: an Extendable Set of Programs as an Aid in Teaching, Research and Application), Elsevier Scientific Software, Amsterdam.

13 J. H. M. Wiitvliet, Internal Reports, Computer Department, University of Technology, Eindhoven, $1987,1988$.

14 I. E. Bush, Anal. Chem., 55 (1983) 2353.

15 Th. P. E. M. Verheggen, F. M. Everaerts and J. C. Reijenga, J. Chromatogr., 320 (1985) 99. 